Foro Aequalis Unidad de Ciencia e Investigación 2021

\title{
El desafío de
}

transformar a

Chile en una

sociedad del

conocimiento

Pedro Pablo Rosso

Marcelo Nöel López

Iván Suazo

Jorge Babul

Marcela Arellano

Diego Cosmelli

Vicente Sandoval 


\section{TABLA DE CONTENIDOS}

Introducción

La globalización y la era del conocimiento

Chile en una encrucijada

Los protagonistas de la globalización

Formación de capital humano avanzado en Chile

El programa Mejoramiento de la calidad y Equidad de la Educación Superior MECESUP

Referencias 


\section{INTRODUCCIÓN}

De cara al futuro, en un contexto de incertidumbre social, política y económica, es inevitable preguntarse si la estrategia de desarrollo que nuestro país ha seguido hasta ahora nos permitirá alcanzar las metas de prosperidad e igualdad de oportunidades que anhela un porcentaje mayoritario de los ciudadanos. Nosotros creemos que difícilmente será posible alcanzar ese objetivo a menos que Chile logre transformarse en una "sociedad del conocimiento". Para ello será necesario hacer confluir una serie de factores entre los que destacan una mayor capacidad de cultivar y crear conocimientos y contar con una fuerza laboral que posee una "masa crítica" de lo que se ha denominado "capital humano avanzado".

El economista Theodore Schultz, ya en el año 1960 en la publicación de su artículo titulado Formación de capital mediante la educación, planteaba "la importancia de pensar en la educación como una inversión en las personas y considerar a sus resultados como una forma de capital. Dado que la educación se convierte en parte de la persona que la recibe, nos referiremos a ella como capital humano". Antes de la aparición de este trabajo, el término "capital humano» no tenía cabida en las discusiones sobre políticas educativas. Sin embargo, considerando que el gasto público en educación es una inversión con una alta tasa de retorno, que se correlaciona significativamente con el logro de metas nacionales, el artículo de Schultz motivó numerosos estudios empíricos.

El término capital humano no es unívoco. Para algunos es el conjunto de hábitos, conocimientos, atributos sociales y de personalidad incorporados en la capacidad de realizar un trabajo para producir valor económico (Goldin, 2016). La Unesco, en su Clasificación Internacional Normalizada de la Educación, lo asimila a sus niveles 7 y 8 , vale decir, los estudios de postgrado de nivel de maestría, especialización o equivalente y doctorado (Unesco, 2011). En el caso de un grado de magíster, los programas de estudio suelen tener como principal objetivo impartir competencias académicas o profesionales avanzadas, que conduzcan a un segundo ciclo o a una certificación equivalente. Los programas de este nivel pueden incluir un importante componente de investigación, aunque no otorgan las certificaciones de un doctorado. Por su parte, los programas de doctorado están orientados a estudios avanzados e investigaciones originales. Por lo mismo, solo pueden ser ofrecidos por instituciones de educación superior que hacen investigación en forma intensiva. Se imparten doctorados, tanto en el campo académico, como en el ámbito profesional e industrial y, por lo general, estos programas culminan con la presentación y defensa de una tesis que representa una contribución significativa y original al conocimiento en los respectivos campos disciplinarios o interdisciplinarios.

No obstante, lo anterior, el que una persona tenga el grado de doctor no implica, necesariamente, que 
deba dedicarse solamente a la investigación. Así lo indican los resultados de un proyecto coordinado por la Organización para la Cooperación y el Desarrollo Económicos (OCDE) sobre las actividades laborales de los graduados de esos programas.

Las cifras varían en los distintos países, pero el reporte señala que, en la Unión Europea, el 51 \% de los doctores trabaja en la industria, mientras que tan solo un 38 \% lo hace en instituciones de educación superior ${ }^{1}$.

En Chile, según un estudio de la trayectoria laboral de los graduados de programas de doctorado beneficiados por becas gubernamentales, el 74,4 \% de ellos trabaja en la educación superior, el 3,9 \% en empresas, el 3,1\% en instituciones del gobierno y el resto en una miscelánea de otras instituciones (CONICYT, 2018).

Brunner y Elacqua (2003) en su Informe de Capital Humano en Chile denominan capital humano avanzado a todos los graduados de la educación superior en sus distintos niveles (técnico, profesional, magíster y doctorado). En cambio, CONICYT utiliza esa denominación para las personas capaces de desempeñar tareas de investigación. Por otra parte, un panel de evaluadores de los programas de formación de capital humano de CONICYT usó la definición siguiente: «profesionales que cuenten con las competencias necesarias para realizar o liderar actividades de investigación, desarrollo y/o innovación basada en ciencia y tecnología; así como, profesionales con capacidades para el desarrollo de políticas públicas» (CONICYT, 2013).

En el presente documento el término «capital humano avanzado» se utilizará para designar a las personas que han recibido el grado de doctor, que es el máximo otorgado por las universidades. Aun reconociendo el hecho de que muchos investigadores no lo tienen, un doctorado certifica que el graduado ha demostrado poseer los conocimientos y competencias que le permiten desempeñarse como investigador independiente y generar conocimientos. Desde una perspectiva de sociedad y desarrollo nacional, ese tipo de competencias reviste una gran importancia porque, como se verá más adelante, para un país, la mayor posibilidad de generar conocimientos se asocia significativamente con bienestar y niveles más altos de desarrollo humano.

Este trabajo fue realizado para analizar la situación en la que se encuentran las universidades chilenas con respecto a la capacidad de formar doctores, para, con base en los resultados y conclusiones, desarrollar propuestas de políticas públicas.

${ }^{1}$ Eurostat. Statistics Explained. «People who have a tertiary education and work in a science and technology occupation, 2012 and 2017». Recuperado de: https://ec.europa.eu/eurostat/statistics- explained/index.php/R_\&_D_personnel. 


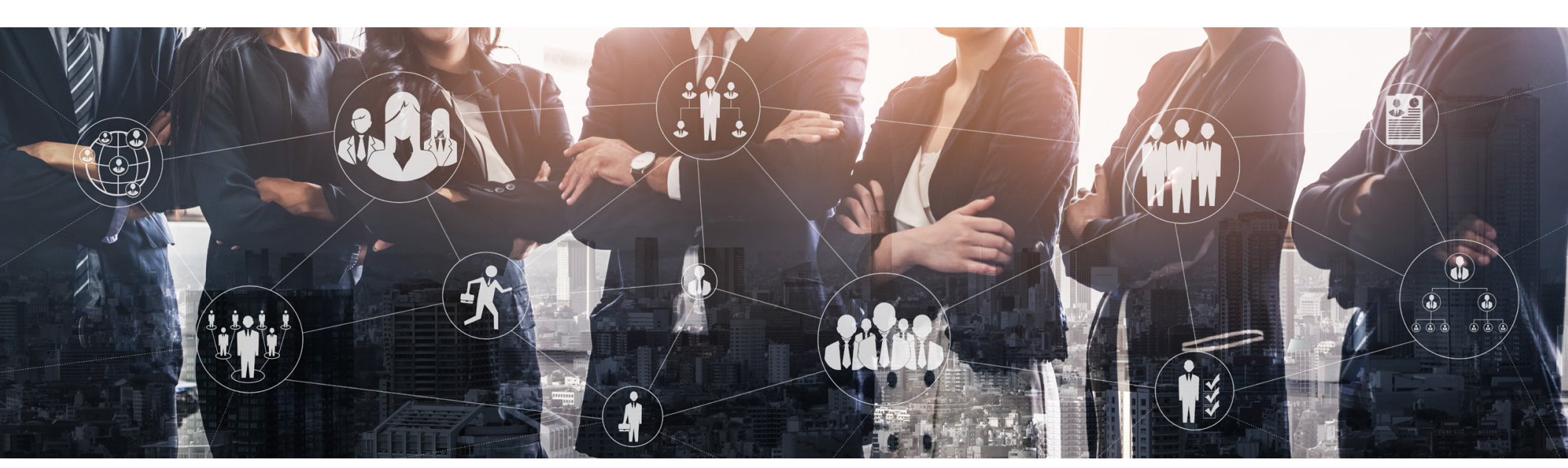

\section{La globalización y la era del conocimiento}

El término «globalización» es el resultado de la evolución semántica del concepto de «mercado mundial», usado inicialmente por Engels a mediados del siglo XIX (Mandel, 1972). En la actualidad, sin embargo, por globalización se entiende un proceso bastante más complejo de integración mundial en los ámbitos económico, social, cultural y tecnológico, lo que ha convertido a nuestro planeta en un lugar cada vez más interconectado (Martell, 2017).

Las determinantes de la globalización han sido diversos elementos interdependientes que han tenido como eje el creciente comercio internacional. Estos incluyen, entre los hechos más significativos, el término de la Guerra Fría o, como lo define Alain Touraine, de la guerra a la sociedad (Touraine, 2005); la consolidación del sistema capitalista, entendido como una organización económica y social; los avances tecnológicos - particularmente en las comunicaciones y la informática - y la disminución de las barreras aduaneras (Martell, 2017). La conjunción de estos factores explica el aumento del comercio internacional, de las transacciones financieras intercontinentales y la expansión de negocios que antes estaban limitados por distancias geográficas o tensiones políticas. La globalización ha modificado notablemente la interacción de las economías nacionales y regionales, promoviendo el crecimiento económico, las democracias de estilo occidental y entregando espacios de mayor prosperidad y bienestar para millones de personas.

Sin embargo, la distribución de la nueva riqueza presenta claras asimetrías y los países exportadores de productos industriales de alta tecnología son más favorecidos si se compara con aquellos exportadores de materias primas y productos semielaborados (CEPAL, 2001). Mientras los primeros tienen como ventajas competitivas la calidad e innovación de sus mercancías y servicios, los segundos basan su 
competitividad en el precio de sus productos y en el menor costo de las remuneraciones. Nuestro país pertenece a este último grupo, junto con las naciones categorizadas como «en vías de desarrollo» que, en general, encuentran en la explotación de sus recursos naturales el sustento de su economía. En cambio, los países del primer grupo o «desarrollados», tienen una larga tradición en la industria de las manufacturas. Paradigmas de ese modelo de desarrollo industrial es Estados Unidos -la mayor economía global actualmente- y los países de Europa occidental, entre los que destacan Alemania, Inglaterra y Francia. Estas economías - a las que se sumó Japón, a partir de los años setenta del siglo pasado, y más recientemente Corea del Sur y China - han sido protagonistas del comercio mundial desde la época de la primera revolución industrial. El largo y sostenido liderazgo económico de estos estados enseña que la capacidad de generar nuevo conocimiento y transformarlo en valor para la sociedad es el principal factor competitivo y fuente de riqueza de las naciones. Por lo mismo, los países que comparten esas características han sido llamados «economías del conocimiento» y, en términos más generales, «sociedades del conocimiento».

Además del éxito económico, existen otros criterios para reconocer una sociedad del conocimiento. En el presente trabajo se considera como tal a una sociedad que valora el conocimiento como activo fundamental para el progreso integral y que centra sus esfuerzos en facilitar que todas las personas puedan acceder a él, potenciarlo, difundirlo e intercambiarlo. En ellas, los sistemas educativos cumplen cabalmente con su misión

fundamental de asimilar, transmitir y crear conocimientos, como también en cada ámbito de la sociedad, donde hay un número significativo de personas - participando en los procesos de toma de decisiones - que son capaces de investigar, plantearse preguntas originales y proponer soluciones a diversos problemas sobre bases racionales y, cuando es posible, basadas en evidencias. Estas personas son el capital humano avanzado de las sociedades del conocimiento. Casi sin excepción, todas ellas han cursado estudios superiores y, en una alta proporción, han obtenido los grados máximos universitarios. La misión de esos programas de estudios, especialmente los doctorados, es profundizar conocimientos en un área específica y, además, aportar aquellas competencias que permiten enfrentar un problema, de cualquier índole, de manera racional, crítica, sistemática e, idealmente, original y eficiente.

Una de las características más notables de la sociedad del conocimiento es el flujo de información de índole artística, humanística y científica que la educación superior transfiere a la sociedad, (Gobierno Vasco, 2010). Este hecho se manifiesta de muchas maneras y de distintas formas, pero una de las más significativas es el uso del conocimiento para el diseño, puesta en práctica y evaluación de las políticas públicas. Este aspecto ha motivado una reflexión sobre la gravitación de los conocimientos en el poder político, ámbito en el cual destacan, por ejemplo, los aportes de Innerarity (2013). Para este autor, en las sociedades del conocimiento, las decisiones que se toman suelen ser producto de una construcción 
social, con la participación de varios actores que han alcanzado una madurez tal en el conocimiento de un área determinada como para ser conscientes de las limitaciones que podría tener su aplicación. Al respecto, Beck (1986) plantea que la ciencia contemporánea ha dejado de ser generadora de certidumbres y, por lo mismo, las personas con conocimientos científicos saben que el progreso no consiste simplemente en acumular y aplicar conocimientos, sino en saber abordar sabiamente la incertidumbre que puede acarrear una decisión de base científica. Esta aceptación de lo desconocido y la disposición a "gestionar el riesgo» y a tomar decisiones sobre bases probabilísticas, no significa que las sociedades contemporáneas desconfíen de las ciencias o dependan menos de ellas, sino todo lo contrario, en este nuevo contexto, la necesidad de contar con una «masa crítica» de capital humano avanzado se torna crucial. Dichas personas, conocedoras de lo que se sabe y lo que se desconoce en una determinada disciplina, son indispensables en la discusión de políticas públicas, muy especialmente cuando los

elementos científicos que las inspiran son debatibles.

Tal como señalan David y Foray (2002), la importancia estratégica del conocimiento y la innovación no es un fenómeno nuevo. Desde las corporaciones de la Edad Media hasta las empresas internacionales surgidas a inicios del siglo $\mathrm{XX}$, los casos de éxito comercial y prosperidad nacional se han asociado a la capacidad de generar conocimientos y usarlos para mejorar la eficiencia de los procesos productivos y la calidad de los productos. Por lo tanto, la principal novedad asociada a la globalización ha sido la de incrementar considerablemente la competencia entre los países y con ello forzar una aceleración del ritmo innovador, y, por consiguiente, de la producción de conocimientos científicos y tecnológicos útiles al sector productivo y de servicios. Paralelamente, la disponibilidad de recursos naturales ha ido perdiendo la importancia que tuvo anteriormente para las economías nacionales, y es reemplazada, en gran medida, por el capital intangible del conocimiento y la capacidad de las personas para crearlo. Por esta razón, la época actual ha sido bautizada como la «era del conocimiento». 


\section{Chile en una encrucijada}

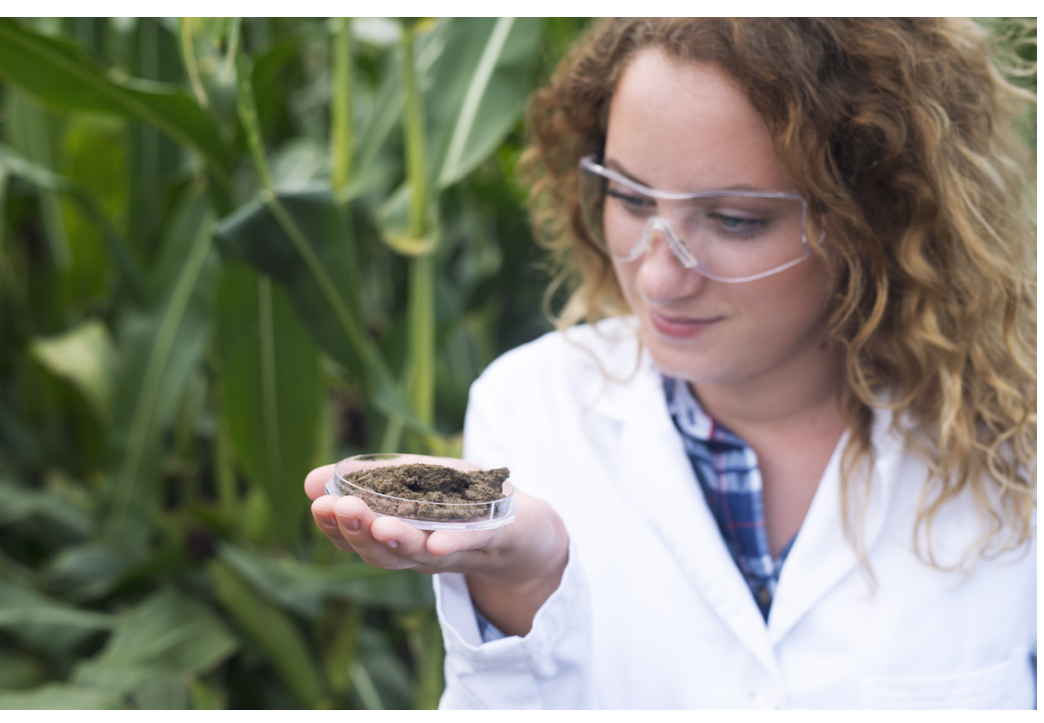

En sus discursos y anuncios, las autoridades de sucesivos gobiernos han planteado la gran tarea de transformar a Chile «en un país plenamente desarrollado», destacando la importancia de la generación de conocimiento y de la innovación para la consecución de ese objetivo. En su programa de gobierno, Michelle Bachelet (2016) planteó la necesidad de impulsar «un fuerte incremento en el fomento a la ciencia, la investigación y la formación de recursos humanos avanzados» y «generar incentivos para que los actores del sistema incrementen sus esfuerzos en materia de innovación y

los orienten hacia objetivos relevantes de desarrollo nacional». Lo anterior con miras a lograr «que Chile se convierta en un país en que el conocimiento y la innovación sean palancas reales de un desarrollo dinámico,inclusivo y sustentable».

Por su parte, el actual mandatario, Sebastián Piñera, enfatizó la necesidad de «sentar las bases para transformarnos en una sociedad de innovadores y emprendedores basados en el uso de nuevas tecnologías, crear las condiciones para que Chile pueda insertarse exitosamente en la cuarta revolución industrial, a partir del desarrollo de nuevos modelos de producción basados en la economía del conocimiento, dar un fuerte salto en materia de ciencia y digitalizar nuestra economía como fuente de mayor productividad y de mejor calidad de vida para los ciudadanos» (2018).

Pero a lo enunciado en ambos gobiernos no ha seguido una estrategia de carácter nacional. Creemos que, para cumplir con esas promesas, se requiere de la elaboración de un plan nacional de ciencia, tecnología e innovación, que defina objetivos, plazos, instrumentos y las inversiones necesarias para cerrar las brechas actuales. De hecho, excepto por la reciente creación del Ministerio de Ciencia, Tecnología, Conocimiento e Innovación, cuya presencia aún no se ha dejado sentir con respecto a las "macropolíticas" de Estado, el panorama de la ciencia y la innovación ha permanecido prácticamente estático durante la última década. Lo anterior ha movido a la comunidad científica a manifestar públicamente, y en reiteradas ocasiones, su preocupación y desconcierto (Hidalgo y otros, 2019).

De acuerdo con Global Competitiveness Report 2019, que valora la capacidad de innovación de los 
países, Chile muestra indicadores descendidos en innovación en el mercado laboral, adopción de tecnologías de información y comunicación, educación, habilidades y salud, que son los que empeoran su capacidad de innovación respecto a los datos de 2018. La más baja performance de Chile está relacionada al ecosistema de innovación.

Hace medio siglo, una estrategia de desarrollo nacional basada en una fuerte inversión en educación y ciencia podría haber conducido a Chile por el camino que ha recorrido Corea del Sur o Singapur. Hoy, una alternativa posible sería intentar un esquema de desarrollo en las líneas del «modelo australiano». Australia es un país próspero, de 24 millones de habitantes, con un PIB per cápita superior a USD 55 mil y una economía basada en la exportación de productos derivados de la minería y de la agricultura. Otros rubros importantes son el turismo y las finanzas. Por más de dos décadas, esta nación ha mantenido una tasa anual de crecimiento sobre el $3 \%$, periodo en el cual no ha sufrido ningún episodio de recesión. Su actual nivel de inversión en investigación y desarrollo equivale al 1,9% del PIB y, en 2018, esto representó una inversión equivalente a USD 6.528 millones. En el mismo período, la inversión de nuestro país bordeó los USD 900 millones (OCDE, 2018a).

De hecho, destaca de Australia la alta prioridad que tiene la calidad de su sistema educativo. Es particularmente notable el caso de la educación superior, cuyo prestigio ejerce una gran atracción en los estudiantes extranjeros, al punto de constituir una importante fuente de divisas. En consonancia con esta realidad, la educación terciaria, la investigación y la innovación son consideradas cruciales para el futuro del país y, en consecuencia, son actores de primera línea en la estrategia australiana de desarrollo (Dawkins, 2019).

Lo antes descrito contrasta fuertemente con la situación de Chile donde, tanto la educación terciaria, como la generación de conocimiento, no ocupan papeles centrales en una estrategia nacional de desarrollo. Tal como señala el Informe del Consejo de CONICYT 2015-2018 (CONICYT, 2018), para abordar el desafío de una política de formación de capital humano avanzado es necesario, en primer lugar, trabajar sobre la base de la visión de un sistema de ciencia, tecnología e innovación. Ese elemento es indispensable para que exista coherencia general de las políticas orientadas a fortalecer las capacidades científicas, tecnológicas y de innovación y, sobre esas bases, determinar las brechas de capital humano.

Dada la falta de esta mirada, es comprensible el grado de descoordinación, duplicidad de iniciativas e improvisación que ha existido en nuestro país en los ámbitos de las ciencias, de la educación terciaria y de la vinculación entre ambas. Probablemente, las cosas se hubieran hecho mejor de haber existido políticas de Estado en estas materias. Ante la carencia de ese marco programático general, el proyecto 
de desarrollo de nuestro país parece limitarse a «hacer mejor y con mayor equidad, más de lo mismo». En este sentido, en la práctica, las políticas que orientan al sistema de educación superior chileno son las de mejorar su tarea formativa y servir como instrumento de movilidad social. Esta falta de horizonte de mediano y largo plazo, como componente fundamental de un plan nacional de desarrollo, ha resultado llamativa para los expertos de la OCDE. En sus informes sobre la educación superior chilena la han considerado una «tarea pendiente», refiriéndose a este vacío conceptual como: «[la necesidad de] desarrollar una visión integral y coherente para el futuro de la educación terciaria, que oriente las futuras políticas de desarrollo a mediano y largo plazo» (OCDE, 2018a). En consonancia con recomendaciones previas de la misma organización, esas políticas «deben estar alineadas con los objetivos sociales y económicos nacionales. Idealmente, debería ser el resultado de una revisión estratégica nacional sistemática de la educación terciaria y conllevar una declaración clara de los objetivos estratégicos» (Santiago y otros, 2008). La estrategia nacional debiera tener un correlato con el desarrollo de una orgánica que brinde sustentabilidad a la implementación de las políticas públicas nacionales.

En los últimos 30 años, algunos de los pasos que ha dado Chile en el ámbito del desarrollo científico incluyen la creación en 1991 del Programa FONDEF de CONICYT, la creación en 2004 del Fondo Innova de CORFO, del Consejo Nacional de Innovación el 2005, el nacimiento de INAPI en 2009, la promulgación de Ley Incentivo I+D en 2012 y la creación, en 2018, del Ministerio de Ciencia, Tecnología, Conocimiento e Innovación, que redunda en que finalmente CONICYT (MINEDUC) derive en ANID para administrar y ejecutar los programas e instrumentos destinados a promover, fomentar y desarrollar la investigación en todas las áreas del conocimiento, el desarrollo tecnológico y la innovación de base científico-tecnológica, de acuerdo a las políticas definidas por el Ministerio de Ciencia, Tecnología, Conocimiento e Innovación. Lo que ha faltado en todas estas iniciativas ha sido, precisamente, una coherencia estratégica y su articulación, por un lado, con un sistema de educación fortalecido desde sus bases y, por otro, con una política nacional de ciencia, tecnología e innovación que trascienda a los gobiernos de turno.

Adicionalmente a lo anterior, sin duda, una de las manifestaciones más patentes y lesivas de lo antes expuesto es la insuficiente inversión pública y privada en ciencia e innovación financiamiento y la baja capacidad de nuestro sistema de educación superior para formar capital humano avanzado. Esto ha sido destacado por expertos de la OCDE en el documento Revisión de Políticas Nacionales de Educación. La Educación Superior en Chile, de 2009, donde se señala la carencia de investigación y desarrollo de alta calidad, y el bajo nivel de inversión en investigación y en políticas educativas. 


\section{Los protagonistas de la globalización}

Actores principales en la era de la globalización y del conocimiento han sido las empresas transnacionales o multinacionales. Hasta fines del siglo pasado, encabezaban este grupo las grandes compañías energéticas, manufactureras y bancarias, pero, evidenciando el dinamismo de la globalización y la importancia de la tecnología de la información, durante el presente siglo, esas empresas han cedido el liderazgo a aquellas del rubro informático, encabezadas por Apple, seguida por Microsoft y Facebook. A este grupo también pertenece Amazon, la empresa líder de las ventas online (Standard \& Poor's Financial Services, 2019).

Sin perjuicio de que los gigantes tecnológicos antes mencionados y otras grandes multinacionales -notablemente, la industria farmacéutica - cuentan con sus propios centros de investigación y desarrollo, los pilares que sostienen a las economías del conocimiento son los sistemas educativos de los respectivos países, que forman a sus fuerzas de trabajo. En ellos, los sistemas educativos son uno de los grandes ejes de los programas de desarrollo, comenzando con una matrícula muy alta en la educación pre-básica. La educación superior tiene un papel estratégico fundamental, por una parte, porque forma los maestros del sistema pre-escolar y escolar básico y medio y, por otra, por su capacidad de generar y transferir conocimientos y formar profesionales y capital humano avanzado. Esta realidad se expresa en el aforismo de Altbach (2005): «No existen países de clase mundial sin universidades de clase mundial» y es refrendado por las ubicaciones de liderazgo que ocupan, en los rankings mundiales, las instituciones de educación superior de las «economías del conocimiento».

Ya sea mediante alianzas con instituciones de educación superior o centros de investigación no universitarios, incluyendo los propios, las empresas o sectores productivos de las economías del conocimiento reinvierten un porcentaje significativo de sus utilidades en el financiamiento de actividades de investigación orientadas a la innovación. A esto se suma la investigación que realizan en esos países las instituciones de educación superior y las entidades estatales de investigación, como el instituto Max Planck en Alemania, el Centro Nacional para la Investigación Científica (CRNS por sus iniciales en francés) en Francia, la Organización de Investigación Científica e Industrial del Commonwealth de Australia o la Academia de Ciencias de la República Popular China, entre otros. En su conjunto, todas estas instituciones e instancias académicas, estatales y empresariales, conforman la plataforma de investigación, desarrollo e innovación con la que cuenta cada «economía del conocimiento».

La importancia y prioridad que las actividades de investigación y desarrollo revisten para los diversos 
países se refleja en el porcentaje del PIB destinado a financiarlas y en el porcentaje de investigadores y capital humano avanzado que integra su fuerza de trabajo en todos los sectores. En el caso de Chile, el cruce de ambos indicadores lo sitúan muy lejos de las "economías del conocimiento». Con una inversión en ciencia y tecnología equivalente al 0,35\% del PIB (2019), nuestro país se ubica en el penúltimo lugar de los países de la OCDE que, en promedio, invierten un 2,38\% del PIB en esas áreas, y a gran distancia de Israel y Corea del Sur, que lideran las inversiones de este tipo con 4,25 \% y 4,23 \% del PIB, respectivamente (Unesco, 2019; Ministerio de Economía, 2019). Igualmente, con solo un investigador por cada mil trabajadores, Chile ocupa los últimos lugares entre los países de la OCDE cuyo valor promedio es de 7,7 investigadores por cada mil trabajadores (Ministerio de Economía, 2019). Esta última cifra es muy preocupante porque, en último término, los auténticos protagonistas de la generación de conocimientos y de la innovación son las personas. La multitud de mujeres y hombres que se plantean problemas, se hacen preguntas y trabajan para responderlas.

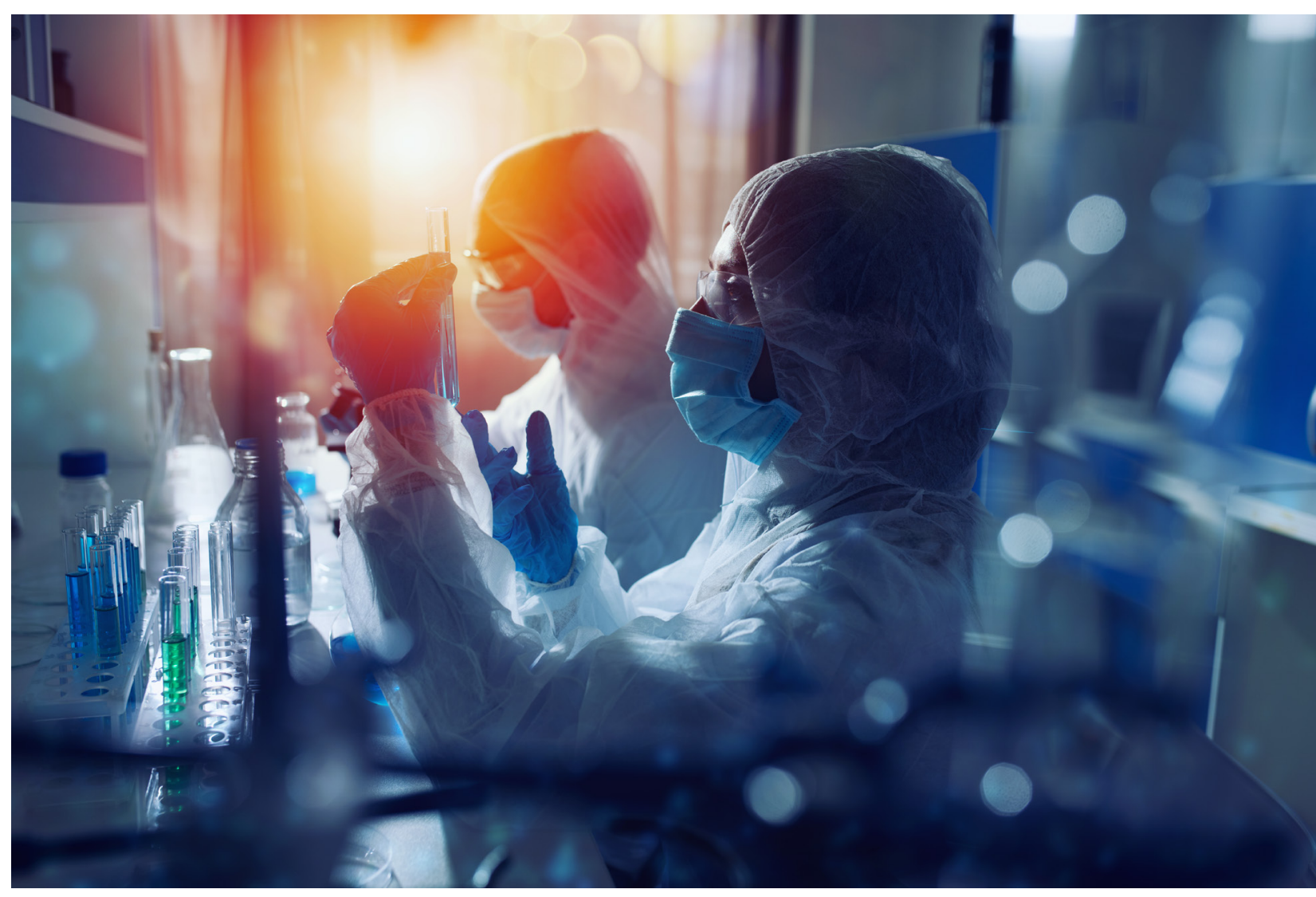


antes de que se iniciara lo que ha sido considerada para nuestro país como la «etapa fundacional» de este tipo de programas, a fines de los años sesenta (Devés y Marshall, 2008; Baeza, 2018). Durante ese largo período de «pausa fundacional» las universidades enviaron a sus académicos a graduarse en instituciones de educación superior de Estados Unidos y Europa occidental.

Según Devés y Marshall (2008), esa primera etapa fundacional transcurre entre 1968 y 1982 , concentrándose la oferta de programas de doctorado en el área de ciencias naturales y exactas (química, física, biología y matemáticas), cuya base académica eran los profesores formados en el extranjero. Hacia el final de esta etapa, el total nacional de candidatos al grado de doctor bordeaba los cien estudiantes (Baeza, 2018).

La segunda etapa histórica de los doctorados chilenos ocupa las décadas de los ochenta y noventa, en el contexto de una gran expansión del sistema chileno de educación superior, y está marcada por el impulso que significaron dos programas de la Comisión Nacional de Investigación Científica y Tecnológica (CONICYT). Estos fueron el Fondo Nacional de Desarrollo Científico y Tecnológico (FONDECYT) y el Programa de Formación de Capital Humano Avanzado" (Becas CONICYT). El primero financiaba proyectos de investigación, mientras que el segundo, mediante los programas Tesis de Doctorado y Término de Tesis de Doctorado, financiaba a estudiantes de doctorado. Entre 1990 y 2000 , CONICYT aportó 588 becas mediante el primer programa mientras que con el segundo pudo financiar 548 becas (en el periodo 1988-1998), todas ellas para programas nacionales, mayoritariamente en ciencias naturales e ingeniería. En esta etapa, la matrícula de los programas de doctorado se expandió de forma muy acelerada, registrándose, en sus inicios, 97 estudiantes y en su culminación 643 (Baeza, 2018).

La tercera etapa de desarrollo, que dura hasta la actualidad, se inició con la puesta en marcha del programa Mejoramiento de la Calidad y de la Equidad de la Educación Superior Chilena (MECESUP), en 1999, y se potenció considerablemente con el Programa "Becas Chile", creado en 2008 (ver más abajo). Durante este lapso, a pesar de que no tenían acceso a fondos del MECESUP, las universidades creadas en los años ochenta, aportaron cuarenta programas de doctorado. De esta manera, demostraron que algunas ya contaban con núcleos de investigadores capaces de sustentar programas de ese tipo. Reflejando este período de crecimiento, que aún no ha perdido dinamismo, la matrícula de los doctorados creció desde setecientos noventa y dos estudiantes en 1999, a más de seis mil en 2019 (SIES, 2019).

\section{EI Programa Mejoramiento de la Calidad y Equidad de la Educación Superior MECESUP}

El programa MECESUP marca un hito en el camino hacia la formación de una sociedad del 
conocimiento. Esta iniciativa, motivada por una visión a largo plazo y caracterizada por una ejecución transparente y de calidad, fue una gran promotora de la creación de programas de doctorado. Mediante sus líneas de incorporación de académicos, creación y ampliación de infraestructura y fortalecimiento y creación de programas, la oferta de doctorados aumentó exponencialmente, duplicándose su número

a nivel nacional en un lapso de 10 años desde su puesta en marcha. Por esta razón, merece una revisión en mayor profundidad.

En 1998, el gobierno de Chile acordó con el Banco Internacional de Reconstrucción y Fomento (BIRF, Banco Mundial), el diseño y puesta en marcha de ese programa. Financiado en parte por el Banco Mundial, tuvo como objetivo central el mejoramiento de la calidad y equidad del sistema de educación superior del país. Durante su vigencia, que comienza en 1999 y llega hasta el presente, esta iniciativa ha tenido tres ciclos. El primero, que cubre el período 1999- 2005, se orientó a la mejora de la infraestructura de las instituciones de educación superior mediante la creación de un Fondo Competitivo. Esto permitió la creación de infraestructura tecnológica, servicios de apoyo a la docencia, servicios estudiantiles diversos, renovación y construcción de bibliotecas y salas de clase, etc. En la gran mayoría de los casos, estos cambios mejoraron considerablemente los estándares de los programas de estudio de pregrado.

El Fondo Competitivo era concursable y sus asignaciones se hicieron considerando, únicamente, los méritos de cada proyecto, lo que representó el abandono de los criterios de asignación históricos, basados en consideraciones de diversa índole y carentes de transparencia. Esto fue una novedad que, inevitablemente, generó algunas tensiones.

Otro logro relevante del primer ciclo fue el diseño e implementación experimental de un proceso voluntario de acreditación institucional y de programas de estudio que, posteriormente, tuvo continuidad en la creación del Sistema Nacional de Aseguramiento de la Calidad.

Del mismo ciclo, también destaca el sistema estricto de rendición de los fondos obtenidos (que contribuyó a instalar una cultura de accountability en el sistema), la expansión del ingreso a estudios superiores de estudiantes provenientes de sectores postergados, el mejoramiento de la calidad y pertinencia de los programas de estudio, el fortalecimiento de la capacidad de gestión institucional y la contratación de académicos con el grado de doctor. Con respecto a esto último, MECESUP favoreció la creación y fortalecimiento de programas de postgrado en áreas de menor desarrollo académico, como las ciencias sociales, educación, artes y humanidades. Durante este lapso, benefició a cuarenta programas de doctorados en funcionamiento, veinticuatro programas en formación y promovió la 
creación de cinco redes de programas. Del total invertido por el Fondo Competitivo en esta etapa, se estima que, en el periodo 1999-2015, aproximadamente USD 100 millones fueron destinados al mejoramiento y fortalecimiento de programas doctorales (Baeza, 2018).

El segundo ciclo abarcó el período $2005-2011$ y su eje prioritario fue la reforma curricular del pregrado

y la innovación en los métodos de enseñanza. Como hecho significativo, inauguró los Convenios de Desempeño en un número limitado de universidades estatales. El Fondo Competitivo fue reemplazado por el Fondo de Innovación Académica (FIAC) como único mecanismo competitivo de asignación de recursos. Entre sus logros se cuenta haber influido apreciablemente a asentar, en la educación terciaria, la cultura de la calidad e innovación, y permitir un escalamiento de los fondos e instrumentos competitivos de asignación de recursos. Entre sus iniciativas menos logradas destaca las dificultades del instrumento para tratar materias institucionales complejas, incluyendo la planificación estratégica, la gestión institucional, el seguimiento y la evaluación de las medidas adoptadas, la evaluación de los resultados del aprendizaje y el seguimiento de los egresados. Con respecto a los programas doctorales, durante este ciclo fueron creados cerca de ochenta programas.

La tercera fase del programa o MECESUP 3 -iniciada en 2012 - tuvo como objetivo mejorar la calidad y la relevancia de la educación superior a través de la ampliación del sistema de financiamiento basado en resultados. Asimismo, pretendió hacer del financiamiento por resultados un rasgo estándar del sistema de financiamiento de las instituciones de educación superior chilenas. En régimen de escalamiento, estuvo orientado a la gestión del cambio en las instituciones de educación superior beneficiadas, enfocándose en tres áreas: a) Mejoramiento del aprendizaje (aumentar en cantidad, calidad y capacidad el capital humano para docencia e investigación) y desarrollo de nuevos programas de estudio en áreas estratégicas; b) Incremento de la eficiencia de la educación terciaria para promover modelos educacionales innovadores, proveer educación remedial a los estudiantes, integrar tecnologías en la experiencia de aprendizaje y facilitar la articulación de la educación técnica con la universitaria; y c) Mejoramiento de la capacidad de gestión institucional.

\section{El programa Becas Chile de CONICYT}

El programa Becas Chile —hasta antes de la creación del Ministerio de Ciencias, Tecnología, Conocimiento e Innovación, su nueva entidad sostenedora- dependió del Ministerio de Educación. Según consta en documentos oficiales, tiene como finalidad «insertar a nuestro país en la sociedad del conocimiento y dar, así, un impulso definitivo al desarrollo económico, social y cultural de Chile» mediante el otorgamiento de becas para realizar estudios y estadías de perfeccionamiento en el extranjero. En la actualidad, con el concurso de proyectos" Becas Chile Crea" también ha ampliado su 
campo de acción al financiamiento de proyectos de formación académica de finalización de doctorado, magíster, diplomados, postítulos, seminarios y congresos.

El programa Becas Chile fue creado en 2008, mediante Decreto Supremo N. 664 del Ministerio de Educación, que señala como su propósito principal «asignar becas de estudio o investigación en todas las áreas del conocimiento y en cualquier país del mundo, excepto Chile». El mismo decreto enumera la variedad de estudios que pueden ser beneficiados por el programa. Estos incluyen doctorado, posdoctorado, magíster, magíster para profesionales de la educación, pasantías doctorales, subespecialidades médicas, pasantías de matemáticas y ciencias y estudios de un semestre en el extranjero para estudiantes de pedagogía en inglés.

Tal como ha sido comentado extensamente en el informe Formación de capital humano en Chile (CONICYT, 2013), la creación de este programa respondió a la necesidad de «poner orden» en el sistema de becas para estudios en el exterior financiadas por el Estado. Antes de 2008, existían las becas Presidente de la República, otorgadas por MIDEPLAN; el programa de becas internacionales de CONICYT; y otros programas de menor envergadura coordinados por el Ministerio de Relaciones Exteriores y el Consejo de la Cultura y de las Artes. A las anteriores, se sumaron las becas de postgrado incluidas en el programa MECESUP. Aparentemente, en la decisión de crear Becas Chile influyeron tres aspectos. En primer término, la necesidad de racionalizar un sistema nacional de becas de postgrado que adolecía de dispersión y descoordinación. En segundo lugar, las recomendaciones del Consejo Nacional de Innovación para la Competitividad, organismo que, en 2007 y 2008, en sendas publicaciones, proponía al Estado avanzar hacia la transformación de Chile en una economía del conocimiento mediante el desarrollo de tres líneas de acción: la formación de capital humano, la ciencia y la innovación empresarial. Por último, el erario fiscal disponía de una importante reserva de divisas provenientes de la minería del cobre.

No obstante, como otra evidencia de la falta de estrategias nacionales respecto a cómo avanzar hacia el «pleno desarrollo», la creación del programa Becas Chile no contó con el respaldo de un documento que presentara, sobre bases empíricas, un diagnóstico de la magnitud y causas del retraso en la formación de capital humano avanzado que el mismo pretendía resolver o aminorar, las razones para la elección de estrategias y sus metas y plazos. Es necesario destacar este hecho porque es un doloroso ejemplo de las políticas públicas basadas en intuiciones y apriorismos que, durante el último medio siglo, han sido la tónica en materias de educación superior; incluyendo la reciente reforma a la educación superior, cuyas dificultades de implementación revelan sus debilidades conceptuales e incongruencias.

Desde la perspectiva de las universidades y comunidades científicas, el aspecto más criticable de 
Becas Chile fue el limitar su campo de acción exclusivamente a la «formación de capital humano en el extranjero». Obviamente, lo categórico de esta afirmación subentiende que, en general, estos son de mejor calidad que los programas nacionales, consideración que, a su vez, abre un abanico de complejas interrogantes. En este sentido, parece legítimo que el Estado quiera complementar la

formación local de doctores como una forma de incrementar su número en un menor plazo, pero, obviamente, fue una decisión tomada sin considerar sus consecuencias para el sistema de educación superior chileno. Por una parte, resulta lógico que exista la posibilidad de formar doctores en el extranjero en áreas que en nuestro país tienen escaso desarrollo. No obstante, financiar estudios en el extranjero en áreas temáticas cultivadas en Chile por programas nacionales de calidad internacional, atenta directamente contra el desarrollo de nuestras universidades y de la ciencia y, por lo tanto, resulta negativo para el desarrollo científico del país.

Otro aspecto que ha suscitado polémica es la ampliación de las líneas de trabajo de Becas Chile con relación a la formación de capital humano avanzado. Aunque la mayor parte de su financiamiento es destinado a programas de magíster y doctorados, es decir a la formación de expertos e investigadores, existe menor claridad sobre la proyección de las otras áreas y perfiles profesionales beneficiados.

Desde el punto de vista de su impacto en la disponibilidad de doctores, las cifras indican que Becas Chile está aportando una proporción significativa de los nuevos graduados. Por ejemplo, en 2015, se graduaron en el extranjero trescientos treinta y siete becarios CONICYT, trescientos ochenta y cuatro becarios CONICYT en programas nacionales y un adicional de trescientos ocho doctores graduados en el país, pero financiados por otros medios.

Pese a que este programa ha generado un número importante de graduados, un informe preparado por la OCDE, en conjunto con el Banco Mundial ${ }^{2}$, revisa la iniciativa señalando en sus conclusiones cuatro preocupaciones relacionadas con el foco y operatividad de dicho programa. Este reporte sugiere un enfoque selectivo acorde con las prioridades establecidas por el Consejo Nacional de Innovación para la Competitividad (CNIC), así como también estrategias para atraer y reinsertar a los graduados a Chile en actividades productivas.

\footnotetext{
2 Banco Internacional de Reconstrucción y Fomento (Banco Mundial) y OCDE (2011). Revisión de Políticas Nacionales de Educación. Programa de Becas Chile. Disponible en: https://bit.ly/2Vqlh7n
} 


\section{Hacia una economía del conocimiento}

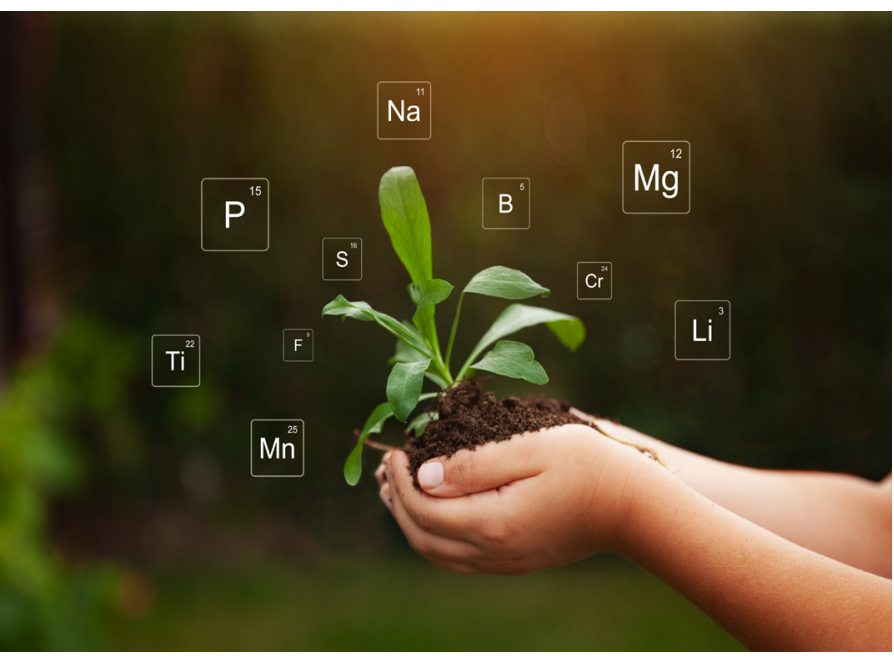

Teniendo presente las enseñanzas que deja la historia de los programas de doctorado en nuestro país y el desafío que implica diseñar y construir un futuro en el que Chile se transforma en una sociedad del conocimiento, surge la pregunta: ¿Cómo se transita desde una sociedad del conocimiento a una «economía del conocimiento»? Se diría que son manifestaciones de un proceso único. Es un cambio de cultura resultante de los conocimientos y competencias que adquieren los ciudadanos en los sistemas educativos. Al respecto, reflexionando sobre la dinámica que impulsa a las economías del conocimiento, Etzkowitz y Leydesdorff (2000) propusieron un modelo que ha tenido gran aceptación e influencia. Inspirándose en la estructura helicoidal del ADN, compuesto por tres elementos de naturaleza distinta, estos autores reseñaron los mecanismos que hacen posible el surgimiento de «economías del conocimiento» como las resultantes de las interacciones mutuas de tres actores: las universidades, el sector industrial y el Estado

Conforme aumentan las interacciones de esa tríada, las instituciones de educación superior (universidades y pokitécnicos) transfiere a la industria los resultados de investigaciones. Esos conocimientos son aplicados para desarrollar nuevos productos y servicios o mejorar la eficiencia productiva. Esto hace más competitivas a las empresas, es decir; más rentables, lo que —a escala nacional y global- determina la prosperidad de esos países. Por su parte, el Estado actúa como catalizador de las relaciones entre las universidades y las empresas, y aporta, para ello, un contexto regulatorio adecuado junto con diversos incentivos. Este sería, por lo tanto, el «ADN» de las economías del conocimiento, una dinámica puesta en marcha en Estados Unidos, durante los años setenta y ochenta, con el surgimiento de la denominada «universidad emprendedora» (Etzkowitz y otros, 2008), vale decir, aquella que interactúa intensamente con el sector industrial y obtiene de ello beneficios académicos y económicos (Clark, 1998). Coincidiendo con este cambio de paradigma, surgió el concepto de la «tercera misión», de las universidades, sumada a las de enseñanza e investigación propias de la universidad humboldtiana (Zawdie, 2010).

Tal como señala González (2009), el núcleo del modelo de la triple hélice es la propagación del conocimiento en la sociedad y la vinculación innovadora de los sistemas de educación superior con el sector empresarial. Esto último posibilita la «capitalización del conocimiento» y, simultáneamente, la «cogniti- 
vización del capital». De ahí que el capital financiero surja sobre el capital social e intelectual acumulado, y que estos se redefinan en tanto las instituciones de educación superior interactúan más intensamente con las industrias y los gobiernos.

Sin desconocer los méritos del enfoque anterior, es necesario aclarar que alcanzar el pleno desarrollo no se limita a un mero proceso técnico-económico para generar prosperidad. Por lo contrario, se trata de lograr un estado en el cual una sociedad, sobre la base de un desarrollo económico exitoso, mejora de manera integral las condiciones de vida de sus miembros. En este sentido, lograr el pleno desarrollo no se limita a asegurar a todos los recursos necesarios para cubrir sus necesidades básicas, sino también significa el acceso a los sistemas de salud y educación, plenas libertades políticas y culturales, así como la posibilidad de cultivar sus intereses y desarrollar sus potencialidades productivas y creativas, entre otras cosas. Desde este enfoque, todo el conocimiento y todos los saberes son importantes para la construcción de ese tipo de sociedad.

Poniendo en paréntesis, para efectos de este análisis, el impacto de la pandemia por SARS-CoV-2, cabe la pregunta: ¿Está Chile en condiciones de poner en movimiento, en las próximas décadas una triple hélice? Lamentablemente, de mantenerse las inercias culturales señaladas en secciones anteriores y el status quo en el ámbito educativo y científico, esa meta parece inalcanzable. Por su naturaleza, la industria chilena ha crecido y prosperado importando tecnología y replicando procesos desarrollados en otros países. Esto vale tanto para las grandes empresas, que emplean un $20 \%$ de la fuerza de trabajo, como para las medianas y pequeñas empresas, que utilizan el $80 \%$ de la fuerza laboral restante. Aunque en Chile existen algunos ejemplos exitosos de innovaciones resultantes de alianzas entre una institución de educación superior y una empresa, son muy pocos los casos que pueden exhibir resultados relevantes y menos aún aquellos con proyección internacional. Un estudio reciente del Ministerio de Economía (2016) analizó la situación de la investigación en las empresas chilenas y concluyó que en este ámbito nuestro país tiene tres problemas básicos: 1) una baja inversión en investigación y desarrollo, 2) un reducido número de investigadores, y 3) un bajo número de doctores. Las cifras que respaldan estas afirmaciones son reveladoras. El aporte del sector empresarial a la inversión en investigación y desarrollo de Chile representa un $33 \%$ del total, comparado con el promedio de los países de la OCDE que aportan un 68 \%. El personal dedicado a actividades de generación de conocimientos e innovación es de 0,42 por cada mil empleados, mientras que el promedio OCDE es de 6,44.

A la desalentadora situación antes descrita se agregan otras complejidades. La primera, de orden probabilístico, se basa en la experiencia mundial relativa a innovaciones exitosas. Esta demuestra que el desarrollo de cualquier producto, sea este un fármaco o un detergente, requiere de una inversión de 
tiempo y dinero considerablemente superiores a las posibilidades económico-financieras y al contexto temporal con que operan las empresas chilenas, particularmente las pequeñas y medianas. En gran medida, lo anterior explica la baja inversión en investigación y desarrollo del sector privado de nuestro país. También aporta luces sobre la situación de desvinculación entre las instituciones de educación superior y empresas, que el Estado no ha podido o no ha sabido resolver. En este ámbito, las iniciativas públicas han sido poco acertadas. Su historial ofrece ejemplos de duplicidad de programas, cambios frecuentes de prioridades, una sostenida baja inversión y algunos desaciertos llamativos. Entre estos últimos, el más notable continúa siendo la ausencia — por disposición reglamentaria- de representantes de las universidades en el Consejo Nacional de Innovación para la Competitividad, un organismo asesor del presidente de la República creado en 2005.

Por otra parte, la situación de la investigación que realizan las universidades tampoco es boyante. Después de tres décadas en las que el sistema universitario ha experimentado una expansión muy significativa, comparativamente, el quehacer científico solo muestra un crecimiento moderado. Como signos positivos, se registra un aumento en el porcentaje de académicos con doctorado contratados por las universidades y una duplicación de la producción científica expresada en la cantidad de publicaciones. Otro avance ha sido el aumento de la asociatividad y las redes de colaboración, tanto las interuniversitarias locales, como aquellas con universidades internacionales. Esto último ha sido favorecido por los contactos y lazos de amistad de los doctores beneficiados con el programa Becas Chile que han regresado al país. Otro factor de incremento en los lazos científicos internacionales ha sido la presencia de los centros con financiamiento basal de CONICYT y los núcleos e institutos Milenio de la Iniciativa Científica Milenio (iniciada originalmente en Mideplan, para luego pasar al Ministerio de Economía, Fomento y Turismo y finalmente al MinCTCI). Algunos de ellos han demostrado una calidad y productividad de alto impacto que resulta atractiva para la comunidad científica internacional.

Cabe destacar, en este contexto, que el indicador que durante las últimas décadas ha experimentado proporcionalmente mayor incremento es el número de las solicitudes de patentes de invención. En 2000, el sistema universitario generó trece solicitudes de ese tipo, mientras que, a partir de 2014, el número anual ha superado las cien, con un máximo de ciento ochenta y uno en 2017.

No obstante, la situación general de la investigación en nuestro país continúa siendo modesta. Por lo menos en relación con los indicadores que ostentan las sociedades del conocimiento. Al respecto, es ilustrativo tener presente la antigua definición de «universidad de investigación» que usaba la Carnegie Foundation. Esta entidad incluía en esa categoría a las instituciones que graduaban anualmente cincuenta doctores en un arco de, a lo menos, quince disciplinas. Según este criterio, en la actualidad, Chile contaría solamente con tres instituciones consideradas de este tipo a nivel internacional: la Universidad de Chile, la Pontificia Universidad Católica de Chile y la Universidad de Concepción. 


\section{El futuro de Chile pasa por un cambio de paradigma}

En el contexto de un mundo globalizado, el gran desafío que enfrenta Chile para alcanzar niveles más altos de desarrollo social y económico es el de transformarse en una sociedad del conocimiento. Este cambio de paradigma implica, como uno de sus elementos axiales, mejorar considerablemente la calidad del sistema educativo, en todos sus niveles. Paralelamente, es necesario ampliar la capacidad científica del país, particularmente a nivel de la educación superior, con dos finalidades: generar conocimientos que posibiliten la innovación tecnológica aplicable a la industria y la formación de capital humano, en todas las áreas disciplinarias. El déficit de personas con esos conocimientos y competencias a nivel de las instituciones de gobierno, las empresas y los sistemas educativos constituye una debilidad estructural que mientras perdure impedirá que nuestro país pueda transformarse en una sociedad del conocimiento.

Dado que el capital humano avanzado se forma en las universidades y, concretamente, en los programas de postgrado - particularmente en los doctorados_- es necesario dotar a las universidades con los medios necesarios para crear nuevos programas y ampliar los existentes. Esto solo se puede lograr ampliando la cantidad de investigadores y dotarlos con los medios necesarios para investigar. De ahí, entonces, la necesidad de incrementar fuertemente la inversión en conocimiento, lo que, a su vez, implica invertir en educación superior. La internacionalización es un ámbito que puede potenciar este esfuerzo de dos maneras: a) mediante alianzas estratégicas internacionales en formación doctoral conjunta. Esta modalidad optimiza los recursos y permite la experiencia internacional de los doctorandos; y b) aprovechar el posicionamiento de Chile en Latinoamérica para que se transforme en un polo de atracción de talentos. Como lo muestra la experiencia internacional, dicha atracción es un motor enorme en la potenciación del sistema de ciencia e innovación. Para ello, se debe pensar de manera integral qué significa atraer talento, no solo en términos de becas y programas, sino también de capacidades de acogida, algo que en todos los países que se han transformado en polo de atracción ha sido fundamental para que pueda ocurrir.

En este sentido, son iluminadores los planteamientos del Consejo de CONICYT (2018) cuando indican que: «Para abordar el desafío de una Política de Formación de Capital Humano Avanzado, es necesario, en primer lugar, trabajar sobre la base de una visión de un sistema de ciencia, tecnología e innovación, de modo tal de asegurar una coherencia general de las políticas orientadas tanto a la generación de capacidades científicas y tecnológicas, como de aquellas que promueven las actividades de investigación, desarrollo e innovación propiamente tales, de manera individual o asociativa, ya sea basadas 
sobre las definiciones de las propias comunidades científicas o bien orientadas por desafíos derivados de las diversas preocupaciones de la sociedad».

Creemos que idealmente esa tarea debiera ser parte de un Plan Nacional de Desarrollo, que contemple las políticas de Estado pertinentes, con horizontes de largo y mediano plazo y evaluaciones anuales de sus avances. En esta línea, es necesario articular un sistema nacional de innovación y, por consiguiente, crear una institucionalidad que coordine sus actores. El número reducido de investigadores activos, tanto en el sector empresarial como en las reparticiones estatales, podría alzarse como el principal obstáculo inicial. Una recomendación que se desprende de las experiencias de países desarrollados es la adopción del desafío de vinculación Universidad, Empresa y Estado. Se necesita un cambio cultural desde las instituciones educativas y la alta gerencia de las industrias. Asimismo, se requiere de un Estado que establezca estrategias de largo plazo, que fomente el ecosistema de innovaciones, mediante el desarrollo redes efectivas, e impulsando la descentralización, a través de estrategias que trasciendan la política de un gobierno en particular.

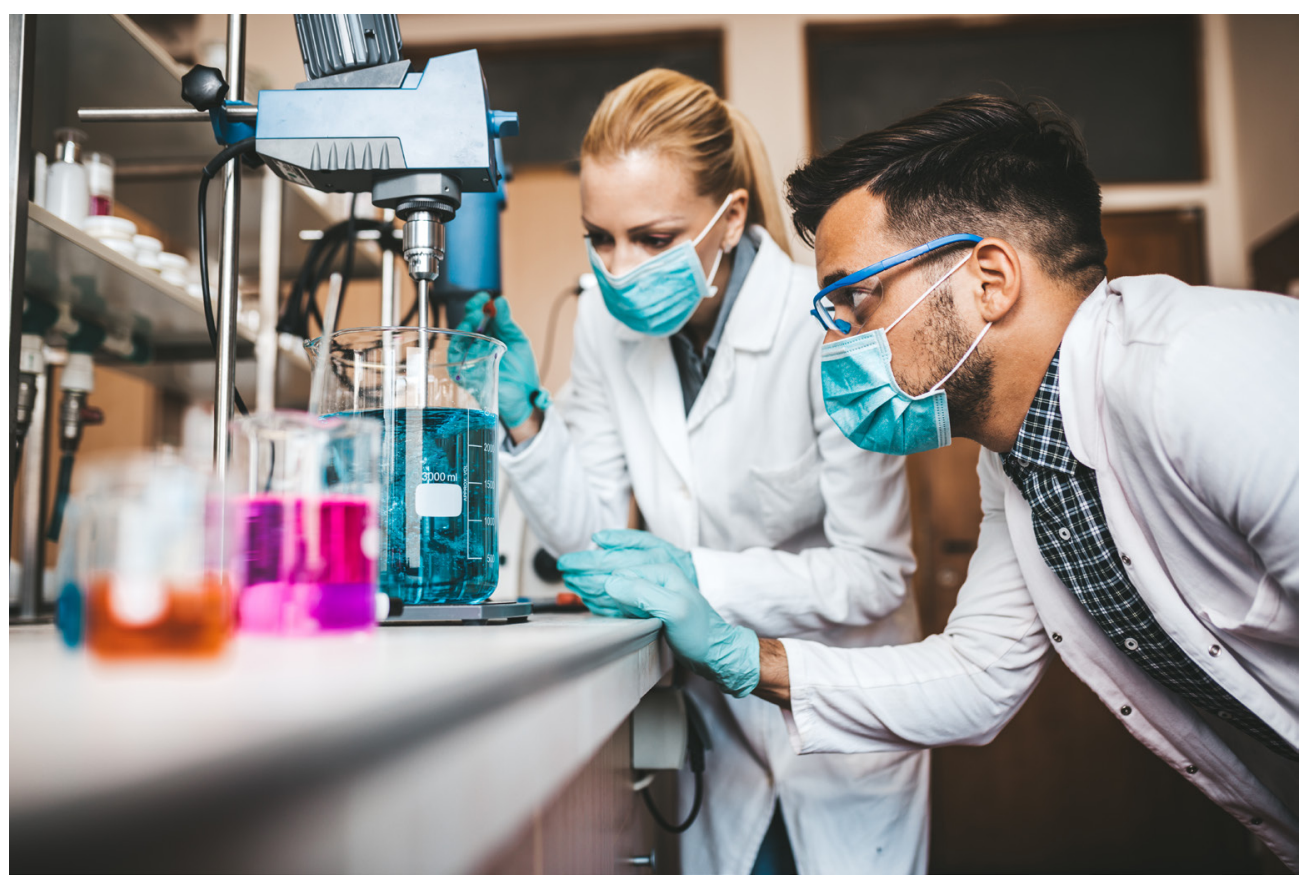

Es importante señalar que esta política de Estado debe articular no tan solo al Ministerio de Educación con el recién conformado Ministerio de Ciencia, Tecnología, Conocimiento e Innovación; para crear un avance significativo hacia una sociedad del conocimiento, por lo que se debe incorporar a los ministerios de Economía, Hacienda y Desarrollo Social.

Asimismo, se debe evaluar una política de fomento de la creación y mejora de programas de doctorado, 
particularmente en las universidades públicas y privadas con sedes regionales, ampliando y diversificando la oferta, que actualmente se concentra en tres universidades (Universidad de Chile, Pontificia Universidad Católica de Chile y Universidad de Concepción).

Finalmente, queremos concluir con la respuesta a una de las preguntas planteadas en nuestras sesiones de trabajo conjunto: ¿Cómo se transita desde una sociedad del conocimiento a una «economía del conocimiento»? Es algo que ocurre en las naciones inevitablemente cuando superan determinados niveles de desarrollo y que es impulsado, indudablemente, por la fuerza del conocimiento que los ciudadanos proponen y que logran traspasar, desarrollar y crear en los sistemas educativos nacionales. En otras palabras, "un país es lo que la gente sabe». 


\section{Referencias}

Altbach, P. G. (2005). «A World-Class Country Without World-Class Higher Education: India's 21st Century Dilema». International Higher Education 40, 18-20. Disponible en:

https://ejournals.bc.edu/index.php/ihe/article/download/7484/6679/

Bachelet, M. (2014). Programa de Gobierno Michelle Bachelet 2014-2018. Disponible en: http://www.subdere.gov.cl/sites/default/files/noticias/archivos/programamb_1_0.pdf

Baeza, P. (2018). «Diversidad y diferenciación en la oferta de programas de doctorado en Chile». Calidad en la Educación 47, 179-214. doi: https://doi.org/10.31619/caledu.n47.34

Banco Mundial (2019). Personas que usan Internet (\% de la población). Disponible en: https://datos.bancomundial.org/indicador/it.net.user.zs?name_desc=fals

Beck, U. (1986). Risikogesellschaft. Auf dem Weg in eine andere Moderne, Frankfurt, Suhrkamp Edición en castellano (1998). La sociedad del riesgo. Hacia una nueva modernidad. Barcelona: Paidós. Citado por Galindo, J. (2015). Acta Sociológica 67, 141-164.,

Brunner, J. J. y Elacqua, G. (2003). Informe Capital Humano. Universidad Adolfo Ibáñez. Disponible en: https://www.oei.es/historico/etp/informe_capital_humano_chile_brunner.pdf\#page31

CEPAL(2002): Globalización y desarrollo. En:

https://repositorio.cepal.org/bitstream/handle/11362/2724/2/S2002024_es.pdf

Clark, B. R. (1998). Creating entrepreneurial universities: Organizational pathways of transformation. Oxford, New York: Published for the IAU Press by Pergamon Press.

CNA, Comisión Nacional de Acreditación (2019). Acreditación de programas de doctorado. Disponible en: https://www.cnachile.cl/Paginas/buscador-avanzado.aspx

CONICYT, Comisión Nacional de Investigación Científica y Tecnológica (2013). Estudio de formación y desarrollo de capital humano. Informe final. Licitación N. ${ }^{\circ}$ 592-130-LP11. Disponible en: https://www.CONICYT.cl/becasCONICYT/files/2015/04/201410141003140.INF_FINAL_FORMACI ON_DSLLO_K_HUMANO.pdf 
(2018). Informe del Consejo de CONICYT 2015-2018. Disponible en:

https://www.CONICYT.cl/wp-content/uploads/2018/03/Interior_CONICYT.pdf

Dawkins, P., Hurley, P., y Noonan, P. (2019). Rethinking and revitalising tertiary education in Australia. Melbourne: Mitchell Institute. Disponible en:http://www.mitchellinstitute.org.au/wp- content/uploads/2019/05/Rethinking-and-revitalising-tertiary-education-FINAL.pdf

Devés, R. y Marshall, M. T. (2008). «El desarrollo del postgrado en Chile». En Brunner, J. J, y Peña, C. (Eds.). Reforma a la educación superior, pp. 265-304. Santiago: Ediciones Universidad Diego Portales.

Etzkowitz, H. y Leydesdorff, L. (2000). «The dynamics of innovation: from National Systems and 'Mode 2 ' to a Triple Helix of university-industry-government relations». Research Policy 29(2) 109-123. Disponible en: https://doi.org/10.1016/S0048-7333(99)00055-4

Etzkowitz, H., Ranga, M., Benner, M., Guaranys, L., Maculan, A. M. y Kneller, R. (2008). «Pathways to the entrepreneurial university: towards a global convergence». Science and Public Policy, 35(9) 681-695. Disponible en: https://doi.org/10.3152/030234208X389701

Funtowicz; S. O. y Ravetz, J, R. (1993): La ciencia posnormal. La ciencia con la gente. ICARIA Antracyt, Centro Editor de América Latina, Buenos Aires. En:

https://economiaecologicaunam.files.wordpress.com/2015/09/2000-funtowicz-y-ravetz-la- ciencia-posnormal.pdf

Gobierno Vasco (2010): Plan Euzkadi en la sociedad de la información. En: https://web.archive.org/web/20100216173522/http://www.innova.euskadi.net/contenidos/info rmacion/pesi2010/es_pesi2010/adjuntos/Plan.pdf

Goldin, C. (2016). «Human Capital». En Diebolt, C. y Haupert, M. (eds). Handbook of Cliometrics. Springer-Verlag. pp. 55-86. Disponible en:

http://scholar.harvard.edu/files/goldin/files/human_capital_handbook_of_cliometrics_0.pdf

González, T. (2009). «El modelo de triple hélice de relaciones universidad, industria y gobierno: un análisis crítico». ARBOR Ciencia, Pensamiento y Cultura. CLXXXV (738) 739-755. Disponible en: doi: 10.3989/arbor.2009.738n1049 
Hidalgo, M.C., Conca, C., Santelices, B., Lagos, R., y Hervé, F. (2019). «Impulsar la generación de conocimientos». El Mercurio, 21 de agosto de 2019, p. 2

Innerarity, D. (2013). «Power and knowledge: The politics of the knowledge society». European Journal of Social Theory. (16) 3-16. Disponible en: https://doi.org/10.1177/1368431012468801

Király G. y Géring Z. (2019): Introduction to 'Futures of Higher Education' special issue. Volume 111, Pages 123-129. Futures, https://doi.org/10.1016/j.futures.2019.03.004

Mandel, E. (1972). El capitalismo tardío. México: Ediciones Era, p.49. Disponible en: https://proletarios.org/books/Mandel-El_capitalismo_tardio.pdf

Martell, L. (2017). The sociology of globalization (second edition). Polity Press: Cambridge.

Ministerio de Economía, Fomento y Turismo (2016). Evaluación de instrumentos de inserción de investigadores en la industria. Informe final. Disponible en: http://ctie.economia.cl/wp- content/uploads/2017/07/Evaluación-de-Instrumentos-de-Inserción-de-Investigadores-en-la- Industria-2016.pdf

Neira, I. (2007). «Capital humano y desarrollo económico mundial. Modelos econométricos y perspectivas». Estudios Económicos de Desarrollo Internacional, 7(2): 53-80. Disponible en: http://www.usc.es/economet/journals/eedi/eedi723.pdf

Organización para la Cooperación y el Desarrollo Económicos, OCDE, y Banco Mundial (2009). Revisión de Políticas Nacionales de Educación, la Educación Superior en Chile. Disponible en: http://www.cnid.cl/wp-content/uploads/2015/10/La-Educaci\%C3\%B3n-Superior-en- Chile_2009.pdf

(2011). Revisión de Políticas Nacionales de Educación. Programa de Becas Chile. Disponible en: http://www.oecd.org/innovation/research/47309025.pdf

Organización para la Cooperación y el Desarrollo Económicos, OCDE (2018a). Educación en Chile. Evaluaciones de Políticas Nacionales de Educación. pp.28. Disponible en: http://archivos.agenciaeducacion.cl/Educacion_en_Chile_OCDE_Nov2017.pdf

(2018b). Economic survey of Australia. Ensuring further success. Disponible en: https://issuu.com/oecd.publishing/docs/ptt_australia_dec_2018/1?ff\&e=3055080/69327326 
(2019). Research and Development Statistics (RDS). Disponible en:

https://www.oecd.org/innovation/inno/researchanddevelopmentstatisticsrds.htm

(2019). OECD work on careers of doctorate holder. Disponible en:

http://www.oecd.org/science/inno/careers-of-doctorate-holders.htm

Piñera, S. (2018). Programa de Gobierno de Chile (2018-2022). Disponible en:

https://observatorioplanificacion.cepal.org/es/planes/programa-de-gobierno-de-chile-2018- 2022

Salas, V., Riveros, M. y Medra, P. (2017). Informe Final de Evaluación. Programas Gubernamentales (EPG). Programas: Becas Nacionales de Postgrado y Becas Chile. Ministerio de Educación. Comisión Nacional de Investigación Científica y Tecnológica. En:

http://www.dipres.gob.cl/597/articles-163122_informe_final.pdf

Santiago, P., Tremblay, K., Basri, E. y Arnal, E. (2008). Tertiary Education for the Knowledge Society. Volumen 1. Special Features: Governance, Funding, Quality. Organización para la Cooperation y el Desarrollo Económicos, OCDE. pp.18-20. Disponible en:

http://www.oecd.org/education/skills-beyond-school/41266690.pdf

Schultz, T. W. (1960). «Capital Formation by Education». Journal of Political Economy. 68 (6) 571-

583. Disponible en: https://www.jstor.org/stable/1829945?seq=1\#page_scan_tab_contents

Sistema de Información de la Educación Superior (2019). Matrícula de estudiantes de programas de postgrado en Chile. Disponible en:

https://www.mifuturo.cl/wp- content/uploads/2019/07/Informe-Matricula-2019_SIES-1.pdf

Standard \& Poor's Financial Services (2019). S\&P 500 Companies by Weight. Disponible en: https://www.slickcharts.com/sp500

Touraine, A. (2005). Un nuevo paradigma para comprender el mundo de hoy. Paidós: Barcelona.

Unesco, Organización de las Naciones Unidas para la Educación, la Ciencia y la Cultura (2011). Clasificación Internacional Normalizada de la Educación. CINE 2011. En: http://uis.unesco.org/sites/default/files/documents/international-standard-classification-of- education-isced-2011-sp.pdf 
(2019): Gasto en investigación y desarrollo (\% del PIB). En:

https://datos.bancomundial.org/indicador/GB.XPD.RSDV.GD.ZS

Välimaa, J. y Hoffma, D. (2008): Knowledge society discourse and higher education. Higher Education 56 (3): 265285.

Zawdie, G. (2010). «Knowledge exchange and the Third Mission of universities». Industry \& Higher Education 24(3), 151-155. Disponible en:

https://pdfs.semanticscholar.org/6a26/b5a8b6ca85bcbccc2f597111305654a1ec34.pdf

Ziman, J. (1996): "'Postacademic Science": Constructing Knowledge with Networks and Norms. Science Studies 9 (1): 67-80. 


\section{Autores}

\section{Pedro Pablo Rosso}

Pedro Pablo Rosso R. Se título de médico-cirujano en 1966 en la Pontificia Universidad Católica de Chile. Entre los años 1963 y 1972 realizó estudios de especialización en pediatría y una estadía de perfeccionamiento académico en la Escuela de Medicina de la Universidad de Cornell, Nueva York, EEUU. En 1972 ingresó al Instituto de Nutrición Humana de la Universidad de Columbia, Nueva York, donde ocupó los cargos siguientes: director del Programa de Magíster en Nutrición (1975-1980), jefe de la División de Crecimiento y Desarrollo (1980-1984), director del Programa de Doctorado (1980-1984) y Vicedirector de ese Instituto (1980-1984). Regresó a Chile, en 1984, incorporándose a la Facultad de Medicina de su alma mater, donde, sucesivamente, fue: Secretario Ejecutivo de la Comisión de Investigación (1984-1991), director del Centro de Investigaciones Médicas (1991-1992) y decano de la Facultad (1992-2000). En el lapso 2000-2010 fue rector de la Pontificia Universidad Católica. Otros cargos: presidente de la Organización de Universidades Católicas de América Latina y el Caribe (2009-2015), y consejero del Consejo Nacional de Educación de Chile (2013-2016). Desde el año 2012 es director ejecutivo del Grupo de Estudios Avanzados Universitas.

\section{Marcelo Noël López}

Marcelo Noël López, es Doctor en Educación Superior en Deustuko Unibertsitatea - Universidad de Deusto, País Vasco, España, Magíster en Gerencia y Políticas Públicas de la Universidad Adolfo Ibáñez e Ingeniero Comercial y Licenciado en Administración y Economía, Facultad de Administración y Economía FAE, Universidad de Santiago de Chile. Además, desarrolló una especialización en el Programa Regional de Formulación y Planificación de Políticas Educativas América Latina y el Caribe, Instituto Internacional de Planeamiento de la Educación IIPE-UNESCO, Buenos Aires, Argentina. Profesor universitario en las áreas de evaluación de impacto educativo, planificación estratégica, evaluación institucional y desarrollo organizacional. Participó en el Programa de Mejoramiento de la Calidad y Equidad de la Educación Superior MECESUP, también en el Sistema de Información para la Educación Superior SIES - Chile y fue Secretario Ejecutivo del Comité de Vicerrectores Académicos de las Universidades del Consejo de Rectores de Chile ( $\mathrm{CRUCH})$, para la implementación del Sistema de Créditos Transferibles SCT- Chile y la movilidad estudiantil universitaria. Actualmente, es Director General de Aseguramiento de la Calidad de la Vicerrectoría de Aseguramiento de la Calidad en la Universidad San Sebastián. 


\section{Iván Suazo Galdames}

Iván Suazo Galdames, es Doctor en Morfología por la Universidad Federal de Sao Paulo (Brasil), Máster en Neurociencias y Biología del Comportamiento por la Universidad Pablo de Olavide (España) y Cirujano Dentista por la Universidad de Chile (Chile). Ha sido profesor de Morfología de Universidades Chilenas y extranjeras, es miembro del grupo de investigación SINPOS (Sistema nervioso periférico y órganos de los sentidos) de la Universidad de Oviedo Tiene más de un centenar de publicaciones en revistas científicas internacionales y ha sido profesor de programas de Master y doctorado en su especialidad. Actualmente es Vicerrector de Investigación y Postgrado de la Universidad Autónoma de Chile y editor en jefe de las revistas European journal of health research y la International Journal of Medical and surgical sciences.

\section{Jorge Babul Cattan}

Jorge Babul es Doctor en Bioquímica (PhD) de la Universidad de lowa, EE.UU., Postdoctorado Departamento de Microbiología y Genética Molecular de la Escuela de Medicina de la Universidad de Harvard, EE.UU. y Químico, Licenciado en Química de la Pontificia Universidad Católica de Chile. Es profesor visitante del Departamento de Bioquímica de la Universidad de lowa, EE. UU. En el Laboratorio Dr. Earle Stellwagen y en el Departamento de Microbiología y Genética Molecular, Escuela de Medicina, Universidad de Harvard, EE.UU. Laboratorio Dr. Dan G. Fraenkel. Actualmente se desempeña como Profesor Titular del Departamento de Biología, Facultad de Ciencias, Universidad de Chile y además, como Director del Programa Académico de Bachillerato, de la Vicerrectoría de Asuntos Académicos de la Universidad de Chile. Ha ocupado numerosos cargos en su trayectoria académica, como Miembro del Consejo de la Sociedad Civil de CONICYT (2017 -2018), Miembro de la Comisión Presidencial Ciencia para el Desarrollo de Chile 2015, Presidente de la Asociación Panamericana de Sociedades de Bioquímica y Biología Molecular, Presidente de la Comisión de Evaluación de la Facultad de Ciencias, Universidad de Chile, Presidente de la Asociación Interciencia, Federación de las Asociaciones para el Avance de la Ciencia en las Américas, Director del Departamento de Pregrado, Vicerrectoría de Asuntos Académicos, Universidad de Chile, Director de la Escuela de Postgrado de la Facultad de Ciencias de la Universidad de Chile, Director Académico, Facultad de Ciencias, Universidad de Chile, Director del Departamento de Química de la Facultad de Ciencias de la Universidad de Chile, Presidente de la Sociedad de Biología de Chile y Presidente de la Sociedad de Bioquímica de Chile. 


\section{Marcela Arellano Ogaz}

Socióloga. Se ha especializado en la detección de áreas sensibles de transformación para la formación técnico-profesional, en el desarrollo de soluciones orientadas al mejoramiento del sistema educacional y en la generación de recursos para las transiciones laborales. Lideró la Reforma Educacional Técnico-Profesional en Chile entre los años 2014-2018; consolidando entre otros el Marco Nacional de Cualificaciones, la Estrategia Nacional de Educación Técnico-Profesional chilena y la Ley que creó Centros de Formación Técnica Estatales. Ha prestado servicios de consultoría para organismos internacionales (BID, FOMIN, Unión Europea, OEI, CINTERFOR-OIT, International Youth Foundation) en diversos países de América Latina, participando además como invitada en eventos y seminarios. Actualmente, es miembro del Directorio del Foro de Educación Superior AEQUALIS y del Comité Consultivo de Formación Técnico Profesional de la Comisión Nacional de Acreditación (CNA), siendo par evaluador de la misma entidad. Se desempeña en la Vicerrectoría Académica del Instituto Profesional Duoc UC como jefa de relaciones institucionales y proyectos académicos.

\section{Diego Cosmelli Sánchez}

Diego Cosmelli es Bioquímico de la Universidad de Chile y Doctor en Ciencias Cognitivas de la École Polytechnique en Francia, con entrenamiento postdoctoral en la Universidad de York en Toronto, Canadá. Es profesor titular de la Escuela de Psicología en la Facultad de Ciencias Sociales de la Pontificia Universidad Católica de Chile, donde desarrolla investigación en psicofisiología humana. Actualmente es Director de la Escuela de Graduados de la Vicerrectoría de Investigación de esta casa de estudios y presidente de la Comisión Asesora de Postgrado del CRUCH".

\section{Vicente Sandoval}

Vicente Sandoval, es Investigador Asociado al Disaster Research Unit de la Universidad Libre de Berlín, Alemania. Doctor (Ph.D.) en Planificación del Desarrollo (University College London, Reino Unido), Máster (M.Sc.) en Gestión Urbana (TU-Berlin, Alemania), y Licenciado en Diseño (UC-Temuco, Chile). Su experiencia es principalmente como investigador de los procesos de desarrollo urbano y desarrollo/cooperación internacional, con foco en la gobernanza urbana del riesgo de desastre, e informalidad urbana y riesgos en Latinoamérica y el Caribe, además de Medio Oriente (Irán y Egipto). Ha trabajado además como investigador y consultor internacional para proyectos con la Comisión Europea, GIZ, y USAID. Desde el 2016, colabora como editor principal de la Revista de Estudios Latinoamericanos sobre Reducción del Riesgo de Desastres (REDER). 